\title{
July 2016 Imaging Case of the Month
}

\author{
Michael B. Gotway, MD \\ Department of Radiology \\ Mayo Clinic Arizona \\ Scottsdale, AZ USA
}

Clinical History: An 18-year-old non-smoking man with a previous diagnosis of Ehlers-Danlos syndrome presented with mild shortness of breath and new cough. Physical examination was normal. The patient was afebrile.

Laboratory data were remarkable except for a mildly elevated white blood cell count of $11 \times 10^{9}$ cells/L. Serum chemistries were within normal limits. Oxygen saturation on room air was $97 \%$.

Frontal chest radiography (Figure 1) was performed.

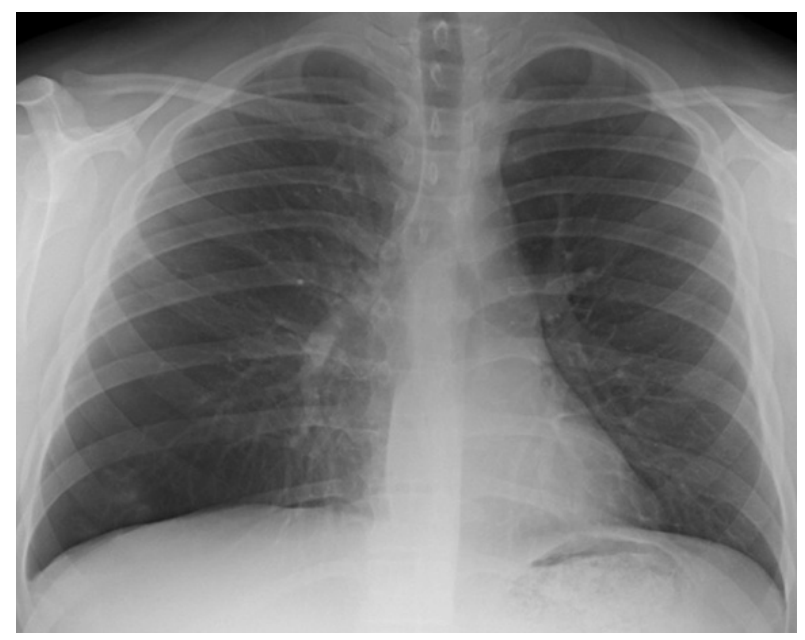

Figure 1. Frontal chest radiography

A previous comparison chest radiograph from 3 years earlier (Figure 2) is shown as well. 


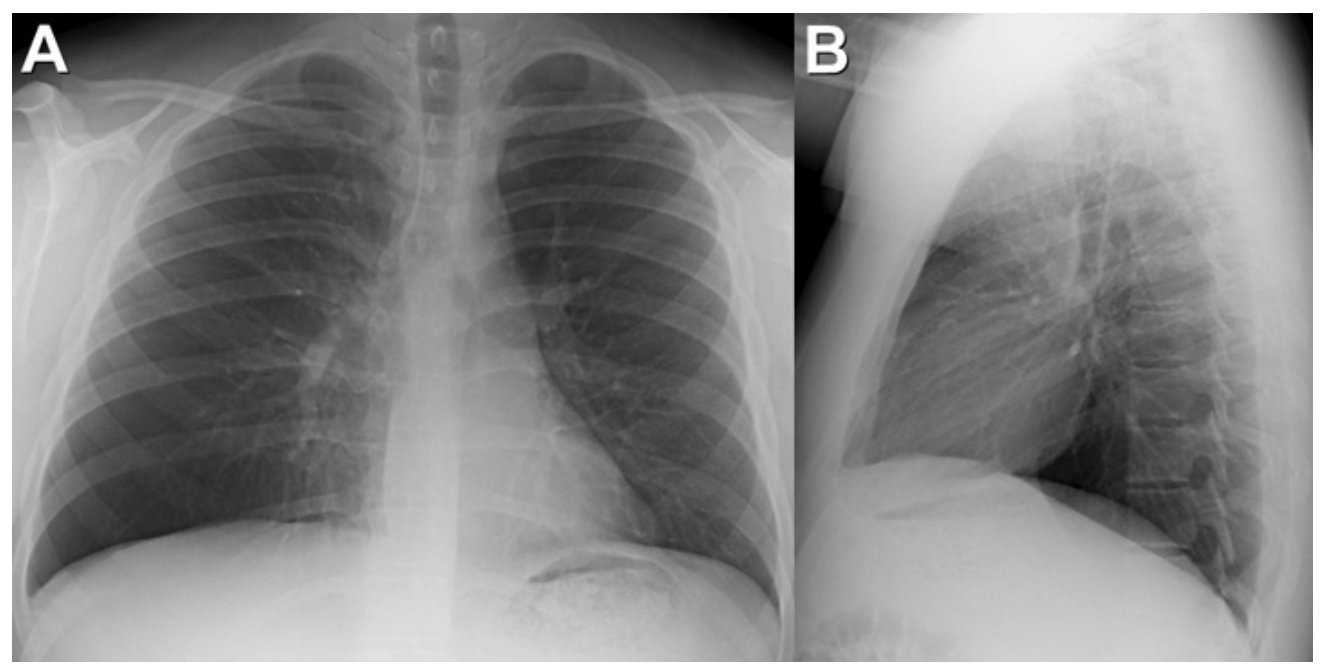

Figure 2. Frontal and lateral chest radiography from 3 years earlier.

Which of the following statements regarding the chest radiograph is most accurate?

1. The frontal chest radiograph shows new bilateral small nodular opacities

2. The frontal chest radiograph shows new focal opacity in the lateral right base

3. The frontal chest radiograph shows new mediastinal lymphadenopathy

4. The frontal chest radiograph shows no focal abnormalities or change from prior

5. The frontal chest radiograph shows pneumothorax 


\section{Correct!}

\section{The frontal chest radiograph shows new focal opacity in the lateral right base}

The chest radiograph shows a new faint nodular opacity in the lateral right base compared with the prior chest radiograph. The mediastinal contours appear normal and no pneumothorax is present. No new bilateral nodules are evident.

The patient was treated for presumed community-acquired pneumonia. Several weeks later, the patient still complained of mild shortness of breath, although the mildly elevated white blood cell count noted at presentation had resolved and his clinical symptoms had improved somewhat. Repeat chest radiography (not shown) appeared relatively unchanged from presentation.

Which of the following is the most appropriate next step for the evaluation of this patient?

1. ${ }^{68} \mathrm{Ga}$-citrate scintigraphy

2. ${ }^{99 \mathrm{~m}}$ Tc-MAA ventilation - perfusion scan

3. Right lateral decubitus chest radiography

4. Thoracic CT

5. Thoracic MRI with contrast - enhanced MR angiography 


\section{Correct! \\ 4. Thoracic CT}

Thoracic CT is appropriate at this point. Thoracic MR would not be of benefit for the evaluation of focal lung opacities. While there are emerging data suggesting that MR of the thorax may be capable of characterizing lung disease, owing to improved scanner technology and faster scan times, the relatively poor signal intrinsic to lung limits the ability of MR to properly characterize pulmonary abnormalities compared with CT. ${ }^{68} \mathrm{Gallium}[\mathrm{Ga}]-$ citrate scanning is occasionally helpful for evaluation of diffuse inflammatory lung disease, but such is not the consideration here. ${ }^{99 \mathrm{~m}} \mathrm{Tc}-\mathrm{MAA}$ ventilation-perfusion scanning is useful the evaluation of suspected acute or chronic thromboembolic disease, but the focal lung opacity in this patient is less suggestive of thromboembolic disease and more consistent with either inflammation or a proliferative process. Decubitus imaging is used for the assessment pleural liquid- to determine of any effusion is free-flowing when the decubitus image is performed on the side with the pleural abnormality- and for assessment of possible air trapping, the latter when the decubitus imaging is performed on the side opposite of the lung suspected of air trapping or endobronchial obstruction. However, these considerations are not relevant for this patient.

The patient underwent enhanced thoracic CT (Figure 3).

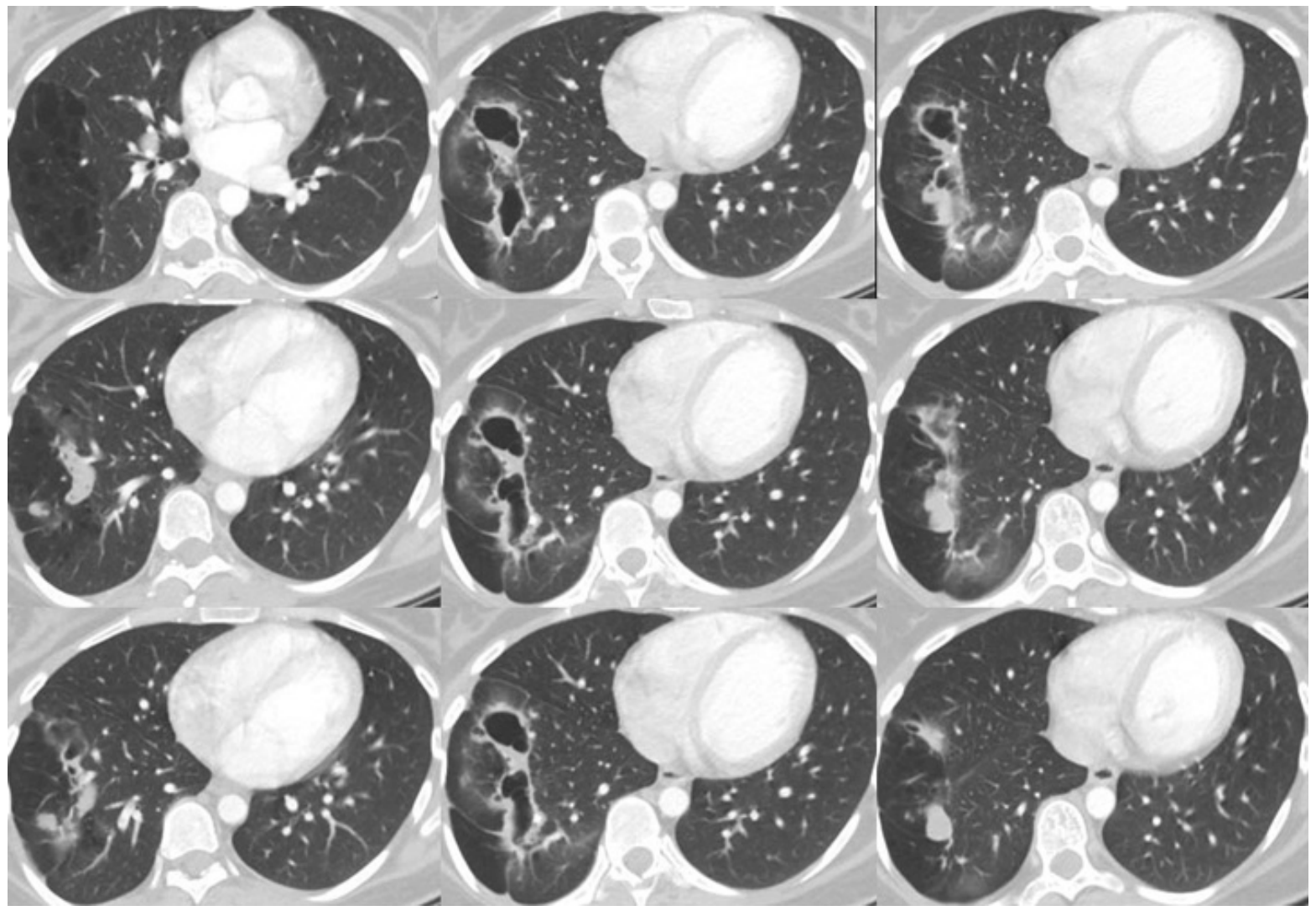

Figure 3. Representative images from the thoracic CT in lung windows. 
Which of the following is correct regarding the description of the thoracic CT findings?

1. Thoracic CT shows bilateral pulmonary emboli

2. Thoracic CT shows focal lucency with solid and cystic opacity in the peripheral right lung

3. Thoracic CT shows lobar consolidation

4. Thoracic CT shows probable hemorrhage around a pulmonary arteriovenous malformation

5. Thoracic CTA shows biapical cavitation 


\section{Correct!}

2. Thoracic CT shows focal lucency with solid and cystic opacity in the peripheral right lung

The thoracic CT shows circumscribed lucency in the right lung, with the inferior medial portion of this lucency showing patchy somewhat tubular opacity as well as more clearly cystic change, possibly even frank cavitation. No pulmonary emboli are seen. The bilateral apices appear normal; no cavitation is evident in these regions. While a somewhat branching appearing opacity is present at the medial right base, no features to suggest an arteriovenous malformation are seen.

Based on the CT appearance, which of the following should be included in the differential diagnosis for the finding at thoracic CT?

1. Carcinoid tumor

2. Congenital pulmonary airway malformation

3. Intralobar sequestration

4. Neurogenic tumor

5. Pneumocystis jirovecii pneumonia 


\section{Correct! \\ 2. Congenital pulmonary airway malformation}

Among the choices listed, congenital pulmonary airway malformation (CPAM) could account for the focal right lung lucency. When CPAMs become infected, some of the cysts can become thick-walled and contain fluid, as is present on the CT. Often CPAM can be associated with bronchial atresia, would could account for the branching, tubular opacity in the medial aspect of the cystic right lung lesion. While cysts may be seen with Pneumocystis jirovecii pneumonia, and some patchy ground-glass opacity surrounds these cysts, the pulmonary findings are essentially unilateral and rather focal, which is inconsistent with Pneumocystis jirovecii pneumonia, and no history of severe immunocompromise, typically present in patients with this infection, is provided. Intralobar sequestration can present with cystic lucency, sometimes appearing more solid when infected, but the key to making the diagnosis of intralobar sequestration is demonstration of the anomalous arterial supply to the lesion, typically from the distal thoracic or upper abdominal aorta, which is lacking in this case. Carcinoid tumor, as well as practically any benign or malignant endobronchial tumor, could produce the branching opacity as well as post-obstructive air trapping; however, the multicystic appearance of the right base lesion is inconsistent with mere overinflation due to air trapping. Neurogenic tumors are exceedingly rare in the lung (typically mediastinal when thoracic in presentation) and appear as solid masses, not multicystic lesions.

The patient was lost to follow up for several years, but then returned for unrelated reasons (foot pain) two years later. A repeat thoracic CT (Figure 4) was performed to follow up the previous abnormalities.

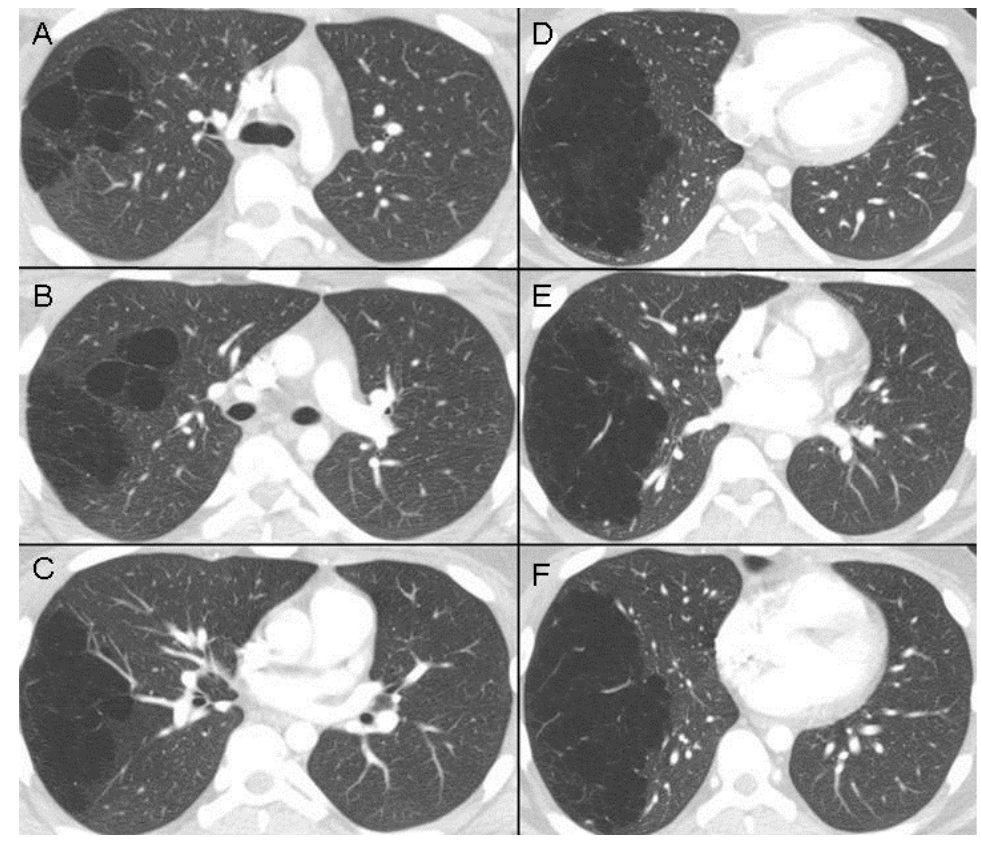

Figure 4. Representative images from an axial enhanced thoracic CT in lung windows performed 2 years later. 
Which of the following is correct regarding this examination?

1. The thoracic CT shows a persistent multicystic process in the right lung

2. The thoracic CT shows complete resolution of the previously noted abnormalities

3. The thoracic CT shows multifocal endobronchial abnormalities with air trapping

4. The thoracic CT shows new multifocal consolidation, now bilateral

5. The thoracic CT shows new peribronchial and mediastinal lymph node enlargement 


\section{The thoracic CT shows a persistent multicystic process in the right lung}

The repeat thoracic $\mathrm{CT}$ shows the same circumscribed multicystic right lung lesion, with resolution of the previous more thick-walled cystic or cavitary appearance inferiorly, with resolution also of the branching solid opacity in the medial right inferior portion of the multicystic lesion. There are no endobronchial opacities or hypolucent foci elsewhere to suggest air trapping, and no new consolidation is evident. The hilar and mediastinal contours appear normal- no lymphadenopathy is evident.

Which of the following represents the next most appropriate step for the evaluation of this patient?

1. Catheter aortography

2. Catheter pulmonary angiography

3. Contrast-enhanced thoracic MRI

4. Mediastinoscopy

5. Resection of the right lung lesion 


\section{Correct! \\ 5. Resection of the right lung lesion}

The multicystic lesion shows no relation to the pulmonary arteries, and no pulmonary artery anomaly, aneurysm, or arteriovenous malformation is seen, so catheter pulmonary angiography is unlikely to be beneficial to the patient. Catheter aortography, particularly for examination of the bronchial arterial circulation and any anomalous systemic arterial vessels supplying the lung, can be useful in some patients, particularly those with intralobar sequestration or inflammatory myofibroblastic tumors, but is typically used to treat such vessels (with embolotherapy) when they have already been identified at contrast-enhanced thoracic CT. In this patient, no such vessels are present, and therefore catheter aortography would not be useful. Contrast-enhanced thoracic $\mathrm{MRI}$ is unlikely to add any additional information to that already known though thoracic CT, particularly for a lung lesion. Mediastinoscopy is primarily used for assessment of mediastinal lesions (paratracheal, anterior subcarinal), either masses, or, more commonly, lymph nodes (in the setting of lung cancer staging), but would not be useful in this patient with a normal-appearing mediastinum and a multicystic lesion in the peripheral right lung. Observation of the lesion could be appropriate, depending on the patient's preferences, but is not offered as one of the choices above..

The patient elected to undergo resection of the right lung multicystic lesion because the lesion was previously infected and there was concern for potential re-infection. Pathological examination showed cystic adenomatoid malformation, with a small focus of bronchial atresia as well

Diagnosis: Congenital pulmonary airway malformation (previously known as congenital cystic adenomatoid malformation)

Post-script: The spectrum if congenital pulmonary airway malformation imaging appearances is presented in Figure 5. 


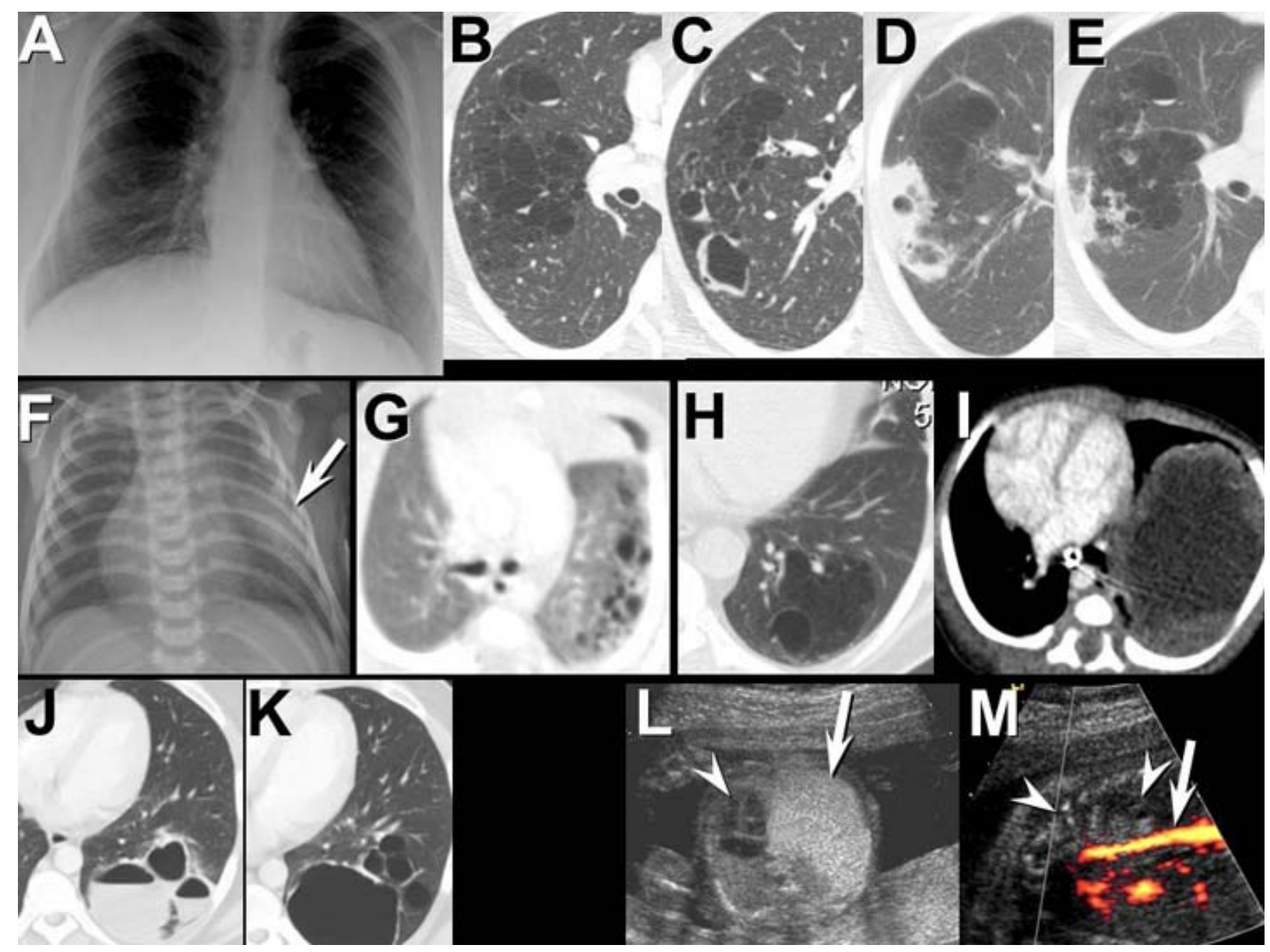

Figure 5. Congenital pulmonary airway malformation: imaging appearances: A-E: Frontal chest radiography $(A)$ in a 30-year-old woman shows faint right upper lobe lucency and nodularity, barely perceptible and non-specific. B and C, axial focused thoracic CT shows a multicystic right upper lobe congenital pulmonary airway malformation. $\mathrm{D}$ and $\mathrm{D}$, Axial focused $\mathrm{CT}$ images at a point when the patient was clinically infected shows patchy consolidation between the cysts and cyst wall thickening. $\mathrm{F}$ and G: Pediatric congenital pulmonary airway malformation: Frontal chest radiography shortly after birth $(F)$ shows a vague, but solid, left upper lung lesion (arrow). Axial thoracic CT performed several days following chest radiography shows a multicystic left lung lesion peripherally, representing a congenital pulmonary airway malformation that has undergone clearing of the intracystic fluid. $\mathrm{H}$ : Congenital pulmonary airway malformation presenting as multiple larger cysts. I: Congenital pulmonary airway malformation presenting as a fluid-filled mass with no gas. $\mathrm{J}$ and $\mathrm{K}$ : Congenital pulmonary airway malformation: infected and uninfected. Axial focused CT

(J) shows multicystic lesion (larger cysts) in the left lung base with several air-fluid levels, representing infection. Note the resolution of the air-fluid levels on the follow up

$\mathrm{CT}$ after treatment of infection $(\mathrm{K})$. $\mathrm{L}$ and $\mathrm{M}$ : prenatal sonography using gray-scale imaging in the axial plane (L) and sagittal power Doppler (MP shows a hyperechoic mass in the thorax (arrow, L) displacing the heart (arrowhead, L). The Power Doppler image shows no evidence of an anomalous systemic arterial supply to the lesion 
(arrow $=$ thoracoabdominal aorta, arrowheads= mass), which favors cystic pulmonary airway malformation over sequestration.

\section{References}

1. Chowdhury MM, Chakraborty S. Imaging of congenital lung malformations. Semin Pediatr Surg. 2015;24(4):168-75. [CrossRef] [PubMed]

2. Gupta N, Vassallo R, Wikenheiser-Brokamp KA, McCormack FX. Diffuse cystic lung disease. Part II. Am J Respir Crit Care Med. 2015;192(1):17-29. [CrossRef] [PubMed]

3. McLoney ED, Diaz PT, Tran J, Shilo K, Ghosh S. Congenital pulmonary airway malformation presenting as unilateral cystic lung disease. Am J Respir Crit Care Med. 2013;188(8):1030-1. [CrossRef] [PubMed]

4. Pacharn P, Kline-Fath B, Calvo-Garcia M, Linam LE, Rubio EI, Salisbury S, Brody AS. Congenital lung lesions: prenatal MRI and postnatal findings. Pediatr Radiol. 2013; 43(9):1136-43. [CrossRef] [PubMed]

5. McDonough RJ, Niven AS, Havenstrite KA. Congenital pulmonary airway malformation: a case report and review of the literature. Respir Care. 2012;57(2):302-6. [CrossRef] [PubMed]

6. Kao SW, Zuppan CW, Young LW. AIRP best cases in radiologic-pathologic correlation: type 2 congenital cystic adenomatoid malformation (type 2 congenital pulmonary airway malformation). Radiographics. 2011;31(3):743-8. [CrossRef] [PubMed]

7. Biyyam DR, Chapman T, Ferguson MR, Deutsch G, Dighe MK. Congenital lung abnormalities: embryologic features, prenatal diagnosis, and postnatal radiologicpathologic correlation. Radiographics. 2010;30(6):1721-38. [CrossRef] [PubMed]

8. Shimohira M, Hara M, Kitase M, Takeuchi M, Shibamoto Y, Kurono K, Shimizu S. Congenital pulmonary airway malformation: CT-pathologic correlation. J Thorac Imaging. 2007;22(2):149-53. [CrossRef] [PubMed] 